\title{
Antiferromagnet-ferromagnet phase transition in lightly doped manganites
}

\author{
I.O. Troyanchuk and V.A. Khomchenko \\ Institute of Solid State and Semiconductor Physics, NAS, 17 P. Brovka Str., Minsk 220072, Belarus
}

E-mail: troyan@ifttp.bas-net.by

\section{V.V. Eremenko and V.A. Sirenko}

B. Verkin Institute for Low Temperature Physics and Engineering of the National Academy of Sciences of Ukraine, 47 Lenin Ave., Kharkov 61103, Ukraine

E-mail: sirenko@ilt.kharkov.ua

\section{H. Szymczak}

Institute of Physics, PAS, 32/46 Lotnikow Str., Warsaw 02-668, Poland

Received February 18, 2005

\begin{abstract}
Magnetic and structural phase diagrams of the $\mathrm{La}_{0.88} \mathrm{MnO}_{x}, \mathrm{La}_{1-x} \mathrm{Sr}_{x}\left(\mathrm{Mn}_{1-x / 2} \mathrm{Nb}_{x / 2}\right) \mathrm{O}_{3}$, $\mathrm{Nd}_{1-x} \mathrm{Ca}_{x} \mathrm{MnO}_{3}$, and $\mathrm{Bi}_{1-x} \mathrm{Ca}_{x} \mathrm{MnO}_{3}$ series constructed on the basis of $\mathrm{x}$-ray, neutron powder diffraction, Young's modulus, magnetization and resistivity measurements are presented. It is shown that the main factor controlling the antiferromagnet-ferromagnet phase transition in the manganites is a type of an orbital state. The results are discussed in the framework of structurally driven magnetic phase separation model.
\end{abstract}

PACS: 75.30.Vn, 75.30.Cr

\section{Introduction}

Mixed-valence manganites with a perovskite structure are the model objects for the physics of strongly correlated electronic systems. The interest in the study of manganites is due to a variety of phase states and transitions and intrinsic correlation of the crystal structure, magnetic, and transport properties. The nature of the interplay between the crystal structure, magnetic, and transport properties of manganites is still a matter of discussion in spite of numerous investigations. Several models were proposed to explain a magnetic state evolution under hole doping as well as a metal-insulator transition at the Curie point. In the double-exchange model of Zener, simultaneous ferromagnetic and metallic transitions have been qualitatively explained by the fact that electrons tend to move between $\mathrm{Mn}^{3+}$ and $\mathrm{Mn}^{4+}$ ions having the same spin orientation, therefore electron delocalization favors the ferromagnetic order [1]. More recently Millis et al. pointed out that double exchange alone cannot account for many of the experimental results [2]. They showed that a Jahn-Teller-type electron-phonon coupling should play an important role in explanation of the colossal magnetoresistance effect. Another mechanism of antiferromagnet-ferromagnet phase transitions in manganites was proposed by Nagaev [3]. He assumed that the intermediate phase can be described as a inhomogeneous magnetic state driven by an electronic phase segregation. In this scenario the ferromagnetic regions contain an excess of holes and are metallic. Goodenough et al. argued that the magnetic properties of manganites were determined by the type of orbital state [4]. According to the rules for $180^{\circ}$ superexchange, if the electronic configuration correlates with vibrational modes, $\mathrm{Mn}^{3+}-\mathrm{O}^{2-}-\mathrm{Mn}^{3+}$ interactions are antiferromagnetic in case of the static Jahn-Teller effect and ferromagnetic when the Jahn-Teller effect is dynamic. Thus, antiferromagnet-ferromagnet phase transitions can occur going through a mixed state of phases with different orbital dynamics. 
The recent magnetic phase diagrams of the $\mathrm{La}_{1-x} \mathrm{Sr}_{x} \mathrm{MnO}_{3}$ and $\mathrm{La}_{1-x} \mathrm{Ca}_{x} \mathrm{MnO}_{3}$ systems were constructed assuming a homogeneous canted magnetic state in a low doping range $[5,6]$. On the other hand, there are numerous experimental data which indicate the existence of phase separation in manganites. The results of nuclear magnetic resonance [7,11], neutron diffraction [11,12], muon spin relaxation [13], x-ray absorption [14], scanning tunneling spectroscopy [15], and electron microscopy [16] experiments give evidence of magnetic and structural inhomogeneities, but the driving force of magnetic phase separation in manganites is still not fully clear. In order to contribute to the solution of this problem we have investigated the features of the antiferromagnet-ferromagnet phase transition in low-doped $\mathrm{La}_{0.88} \mathrm{MnO}_{x}$, $\mathrm{La}_{1-x} \mathrm{Sr}_{x}\left(\mathrm{Mn}_{1-x / 2} \mathrm{Nb}_{x / 2}\right) \mathrm{O}_{3}, \mathrm{Nd}_{1-x} \mathrm{Ca}_{x} \mathrm{MnO}_{3}$, and $\mathrm{Bi}_{1-x} \mathrm{Ca}_{x} \mathrm{MnO}_{3}$ manganites.

\section{Results and discussion}

\section{1. $\mathrm{La}_{0.88} \mathrm{MnO}_{x}$ system}

Tentative magnetic phase diagram of the $\mathrm{La}_{0.88} \mathrm{MnO}_{x}(2.82 \leq x \leq 2.96)$ manganites is shown in Fig. 1. The most strongly reduced sample $\mathrm{La}_{0.88} \mathrm{MnO}_{2.82}$ is antiferromagnet with a Néel temperature of $140 \mathrm{~K}$. Its properties are found to be similar to

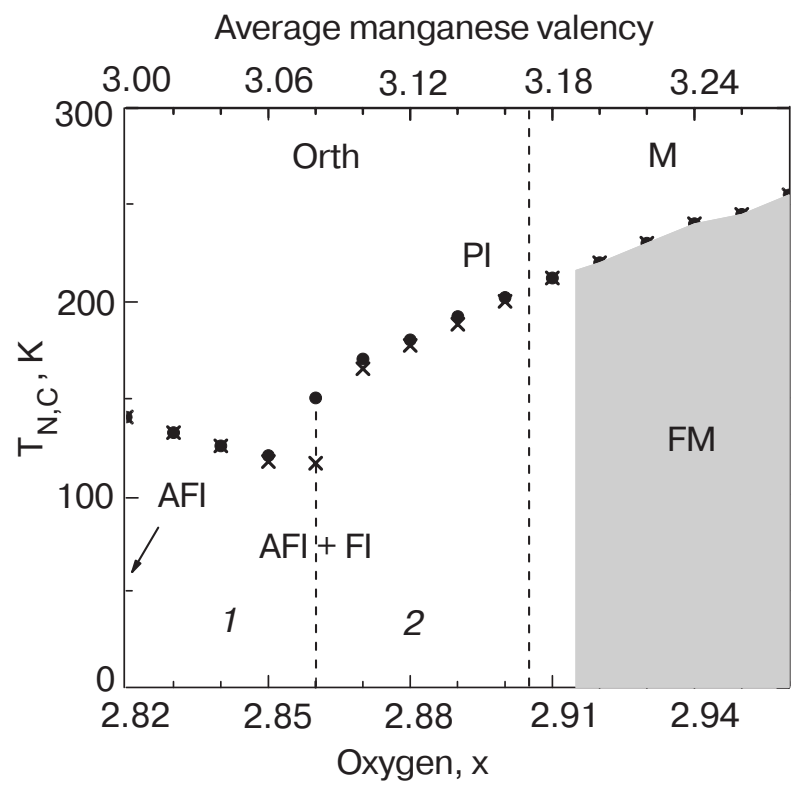

Fig. 1. Magnetic phase diagram of the $\mathrm{La}_{0.88} \mathrm{MnO}_{x}$ $(2.82 \leq x \leq 2.96)$ system. Orth-orthorthorhombic crystal structure, $\mathrm{M}$ is monoclinic crystal structure; PI, AFI, FI, and FM are paramagnetic insulating, antiferromagnetic insulating, ferromagnetic insulating, and ferromagnetic metallic states, respectively. Areas 1 and 2 correspond to the concentration regions where an antiferromagnetic or ferromagnetic phase predominates, respectively. the properties of stoichiometric $\mathrm{LaMnO}_{3}$. Both compounds have very close unit cell parameters, the same magnetization value, and close temperatures of both magnetic $\left(T_{N} \sim 140 \mathrm{~K}\right)$ and orbital orderings $\left(T_{O O} \sim\right.$ $\sim 750 \mathrm{~K})$. The existence of orbital ordering in the $A$-type antiferromagnetic structure of $\mathrm{La}_{0.88} \mathrm{MnO}_{2.82}$ is corroborated by neutron diffraction measurements [17]. With increasing oxygen content up to the $x=$ $=2.85$ sample, the magnetic and orbital ordering temperatures lower while the magnetization increases slightly. Results of the neutron diffraction measurements carried out for the $x=2.84$ sample confirm the appearance of a ferromagnetic component. A further increase of the oxygen concentration leads to a significant enhancement of the ferromagnetic contribution. The transition temperature to the paramagnetic state begins to increase and the transition becomes broader. Neutron diffraction data obtained for the $x=2.87$ sample indicate that ferromagnetic coupling becomes predominant. No long-range antiferromagnetic order has been observed for this compound. At the same time, the refined magnetic moment is lower than that expected for the full spin arrangement. Besides, the relatively large magnetic anisotropy at low temperature assumes the presence of an anisotropic magnetic coupling which differs from the isotropic ferromagnetic one. This can be attributed to existence of either short-range antiferromagnetic clusters or a spin-glass phase. No pronounced thermomagnetic irreversibility indicating the anisotropic magnetic interactions is observed starting from the $x=2.92$ sample. The values of magnetization estimated for the monoclinic compounds are close to those expected for full spin alignment. The ground state of all the orthorhombic compounds $2.82 \leq x \leq 2.90$ is insulating. It should be noted that the appearance of metallic conductivity does not coincide with the transition to monoclinic phase. Simultaneous first-order magnetic transition and metal-insulator transition at $T_{C}$ are observed for $x \geq 2.92$ compounds.

A strong correlation between the magnetic and structural properties of $\mathrm{La}_{0.88} \mathrm{MnO}_{x}(2.82 \leq x \leq 2.96)$ manganites is observed. The hypothetical structural phase diagram of $\mathrm{La}_{0.88} \mathrm{MnO}_{x}(2.82 \leq x \leq 2.96)$ constructed using $\mathrm{x}$-ray, neuron diffraction, Young's modulus, resistivity, and DTA data is shown in Fig. 2. For $\mathrm{La}_{0.88} \mathrm{MnO}_{2.82}$, the sharp anomalies of the Young's modulus and resistivity are associated with the removal of cooperative orbital ordering; it is observed at approximately $650 \mathrm{~K}$. The DTA measurements revealed the release of latent heat in the range $650-730 \mathrm{~K}$. Neutron diffraction data indicate the coexistence of orbitally ordered $\mathrm{O}^{\mathrm{I}}$ and orbitally disordered $\mathrm{O}$ phases at $T=700 \mathrm{~K}$. Another thermal anom- 


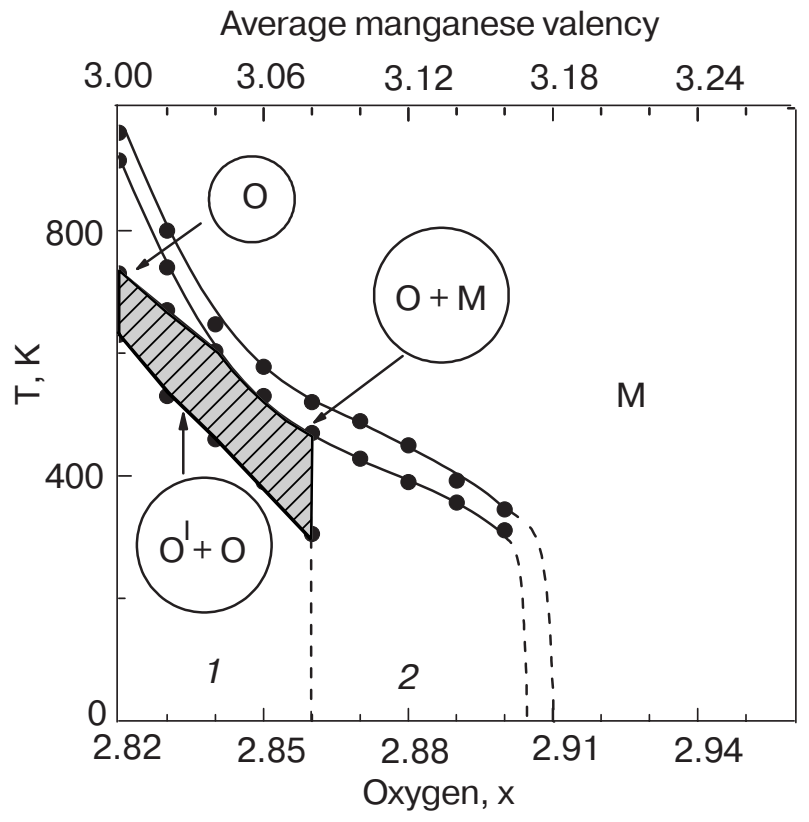

Fig. 2. Crystal structure phase diagram of the $\mathrm{La}_{0.88} \mathrm{MnO}_{x}$ $(2.82 \leq x \leq 2.96)$ system. $\mathrm{O}^{\mathrm{I}}, \mathrm{O}$, and $\mathrm{M}$ are orbitally ordered orthorhombic, orbitally disordered orthorhombic, and monoclinic phases, respectively. Areas 1 and 2 correspond to the concentration regions where the static Jahn-Teller distortions or dynamic orbital correlations predominate, respectively.

aly connected with the transition to the monoclinic phase is observed in the temperature range $915 \leq T \leq$ $\leq 960 \mathrm{~K}$. With the increase of the oxygen content to $x=2.83$, the temperatures of both orbital order-disorder and orthorhombic-monoclinic phase transitions significantly decrease. The range of coexistence of $\mathrm{O}^{\mathrm{I}}$ and $\mathrm{O}$ phases becomes broader, while the width of the anomaly associated with the temperature-induced orthorhombic-monoclinic transition remains practically constant. Starting from the $x=2.84$ sample, the differential thermal analysis does not show any significant heat effect, which could be interpreted as a transition to a pure orbitally disordered state; however, the anomaly related to the transition from an orthorhombic to a monoclinic phase remains well pronounced. Neutron diffraction data coupled with Young's modulus measurements indicate the existence of predominantly static Jahn-Teller distortions at room temperature and two-phase character of the crystal structure above $T \sim 470 \mathrm{~K}$. Inhomogeneous structural states are observed up to $650 \mathrm{~K}$. Above this temperature the monoclinic phase is stabilized. A further increase of the oxygen concentration leads to the broadening and gradual disappearance of the anomaly which relates to the transition to the orbitally ordered state. The neutron diffraction study performed for the $\mathrm{La}_{0.88} \mathrm{MnO}_{2.87}$ compound indicates that the value of the $\mathrm{MnO}_{6}$ octahedron distortion increases with decreasing temperature to $200 \mathrm{~K}$. However, even in the case of $T=200 \mathrm{~K}$, where the worst agreement factors for one-phase structural model have been observed, the introduction of the second orthorhombic phase was unsuccessful. Apparently, even at $200 \mathrm{~K}$, the orbitally ordered clusters are still too small and separate to distinguish the $\mathrm{O}^{\mathrm{I}}$ phase in the diffraction experiment. The temperature of orthorhombic-monoclinic phase transition gradually decreases as the oxygen content increases and starting from the $x=2.91$ sample, the monoclinic phase is stabilized (Fig. 2). It is necessary to mention that the $\mathrm{x}$-ray and neutron diffraction experiments can reveal a two-phase structural state rather in the case of macroscopic structural phase separation. In the cases of local structural inhomogeneities or nanometer scale structural clusters, these experiments give only an average picture of a structural state [18]. Thus, the correlation between the orbital state and magnetic properties of the $\mathrm{La}_{0.88} \mathrm{MnO}_{x}$ manganites is prominent. The static Jahn-Teller distortions are responsible for the $A$-type antiferromagnetic structure, while dynamic orbital correlations lead to ferromagnetism.

It is worth noting that there are two alternative models of orbital state corresponding to ferromagnetic ordering in manganites: $3 D$ dynamic $d_{3 z^{2}-r^{2}}$ orbital correlations and staggered ordering of $d_{3 z^{2}-r^{2}}$ and $d_{x^{2}-y^{2}}$ orbitals. Neutron diffraction studies have shown that $\mathrm{LaMnO}_{3}$ undergoes a structural transition from $\mathrm{O}^{\mathrm{I}}$-orthorhombic to O-orthorhombic phase at $T_{J T}=750 \mathrm{~K}$ [19]. The $\mathrm{MnO}_{6}$ octahedron in the O-orthorhombic phase becomes nearly regular, i.e., the orbital ordering disappears [19]. However, x-ray absorption near the edge structure and the extended $\mathrm{x}$-ray absorption fine structure at the MnK-edge measurements have revealed that the $\mathrm{MnO}_{6}$ octahedrons in $\mathrm{LaMnO}_{3}$ remain tetragonally distorted at $T>T_{J T}$ [20]. The empty $\mathrm{Mn}^{3+}$ electronic $d$-states were shown to be unaltered through the Jahn-Teller transition. The lowest energy for the $e_{g}$ electron corresponds to the three possible distortions giving rise to three degenerate vibronic states, $d_{x^{2}-r^{2}}, d_{y^{2}-r^{2}}$, and $d_{z^{2}-r^{2}}$, being the electronic orbitals of the vibronic state. The thermally excited electron jumps between these states above $T_{J T}$ and is localized in an ordered state below $T_{J T}$. The orbital ordering proposed for $\mathrm{LaMnO}_{3}$ arises then from the ordering of the local Jahn-Teller distortions. The high temperature (O-orthorhombic) phase can be described as a dynamical locally distorted phase with the strong antiferrodistortive first neighbour coupling [20].

The similar situation seems to be observed for $\mathrm{Mn}^{4+}$-doped manganites. The atomic pair-density 
function of $\mathrm{La}_{1-x} \mathrm{Sr}_{x} \mathrm{MnO}_{3}$ manganites $(0 \leq x \leq 0.4)$, obtained by pulsed neutron diffraction, indicates the existence of tetragonally distorted $\mathrm{MnO}_{6}$ octahedrons even in the rhombohedral metallic phase, when the crystallographic structure shows no JT distortions [21]. This is possible only in the case of the dynamic orbital correlations described above. One can assume that when one puts non-Jahn-Teller $\mathrm{Mn}^{4+}$ ions in the background of the $\mathrm{Mn}^{3+}$ ions, the $e_{g^{-}}$-orbitals of all the $\mathrm{Mn}^{3+}$ ions surrounding the localized hole $\left(\mathrm{Mn}^{4+}\right)$ tend to be directed towards it, forming an orbital polaron [22]. Due to the strong antiferrodistortive $\mathrm{Mn}^{3+}$ first neighbour coupling [20], dynamic correlations of the $d_{3 z^{2}-r^{2}}$ orbitals should arise.

According to the rules for $180^{\circ}$ superexchange the dynamic orbital correlations lead to ferromagnetic interaction between the $\mathrm{Mn}^{3+}$ ions [4]. Hence, one can expect that ferromagnetism in manganites can arise even in the absence of $\mathrm{Mn}^{4+}$ ions, if only the JT effect is dynamic. For instance, Mn substitution with non-Jahn-Teller diamagnetic $\mathrm{Nb}^{5+}, \mathrm{Al}^{3+}, \mathrm{Sc}^{3+}$, etc, ions should result in the appearance of ferromagnetic order. Below we show that this assumption is correct.

Second possibility lies in description of orbital state as a hybridization of the $d_{3 z^{2}-r^{2}}$ and $d_{x^{2}-y^{2}}$ orbitals as $\cos (\theta / 2)\left|3 z^{2}-r^{2}\right\rangle \pm \sin (\theta / 2)\left|x^{2}-y^{2}\right\rangle$. Such an orbital ordering is recently proposed experimentally and theoretically in the ferromagnetic insulating phase of $\mathrm{La}_{0.88} \mathrm{Sr}_{0.12} \mathrm{MnO}_{3}$ and $\mathrm{Pr}_{0.75} \mathrm{Ca}_{0.25} \mathrm{MnO}_{3}$ [23-25]. The difficulties in determination of priority of the present models are conditioned by the fact that staggered ordering of $d_{3 z^{2}-r^{2}}$ and $d_{x^{2}-y^{2}}$ orbitals can exhibit itself in experiments in the same way as $3 D$ dynamic $d_{3 z^{2}-r^{2}}$ orbital correlations.

$$
\text { 2.2. } \mathrm{La}_{1-x} \mathrm{Sr}_{x}\left(\mathrm{Mn}_{1-x / 2} \mathrm{Nb}_{x / 2}\right) \mathrm{O}_{3} \text { system }
$$

Hypothetical magnetic phase diagram of $\mathrm{La}_{1-x} \mathrm{Sr}_{x}\left(\mathrm{Mn}_{1-x / 2} \mathrm{Nb}_{x / 2}\right) \mathrm{O}_{3}$ is shown in Fig. 3. The parent $\mathrm{LaMnO}_{3}$ compound shows the spontaneous magnetization value at $5 \mathrm{~K}$ corresponding to magnetic moment of $0.07 \mu_{B}$ per $\mathrm{Mn}^{3+}$ ion. The Neel point where spontaneous magnetization develops is $143 \mathrm{~K}$. According to [26] the spontaneous magnetization has a relativistic nature. Substitution of Mn with Nb leads to an enhancement of the spontaneous magnetization whereas the temperature of transition into paramagnetic state slightly decreases. In accordance with the magnetization data the $\mathrm{La}_{0.8} \mathrm{Sr}_{0.2}\left(\mathrm{Mn}_{0.9} \mathrm{Nb}_{0.1}\right) \mathrm{O}_{3}$ and $\mathrm{La}_{0.7} \mathrm{Sr}_{0.3}\left(\mathrm{Mn}_{0.85} \mathrm{Nb}_{0.15}\right) \mathrm{O}_{3}$ samples are ferromagnets with the magnetic moment per chemical formula around $2.3 \mu_{B}$ and $2.6 \mu_{B}$, respectively. Neutron diffraction study has revealed the magnetic moment of $\mathrm{Mn}^{3+}$ in the parent $\mathrm{LaMnO}_{3}$ antiferromagnetic compound to be close to $3.5 \mu_{B}$ [27] whereas $\mathrm{Nb}^{5+}$ is dia-

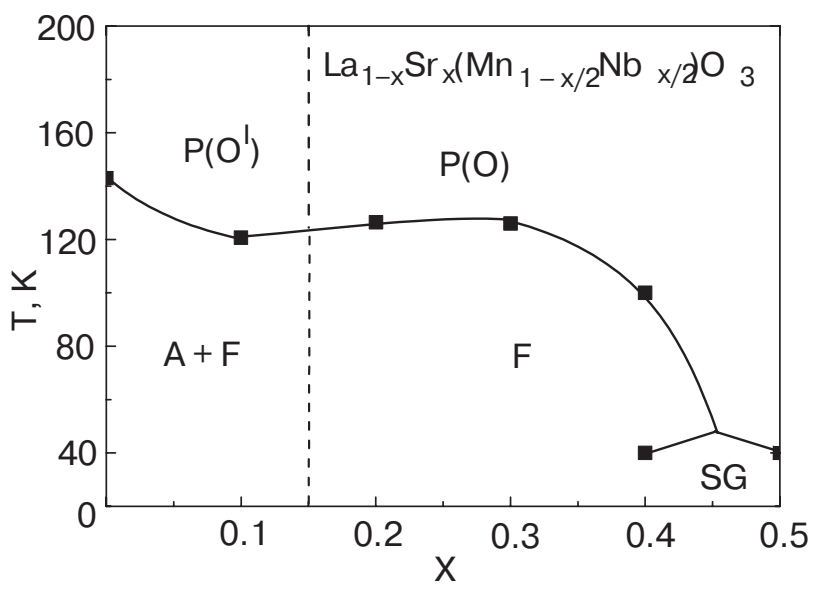

Fig. 3. Magnetic phase diagram for $\mathrm{La}_{1-x} \mathrm{Sr}_{x}\left(\mathrm{Mn}_{1-x / 2} \mathrm{Nb}_{x / 2}\right) \mathrm{O}_{3}$ series ( $\mathrm{A}$ is antiferromagnet, $\mathrm{F}$ is ferromagnet, $\mathrm{P}$ is paramagnet, $\mathrm{SG}$ is spin glass; $\mathrm{O}^{\mathrm{I}}$ and $\mathrm{O}$ is orbitally ordered and orbitally disordered phases, respectively).

magnetic ion, hence the expected moment should be close to $3 \mu_{B}$ per formula unit being in a rather good agreement with the observed one. The $\mathrm{Nb}$ doped sample $(x=0.3)$ has a well defined Curie point $-123 \mathrm{~K}$. Both Curie point and spontaneous magnetization start gradually to decrease when $\mathrm{Nb}$ content exceeds 15\% from total sites number in the manganese sublattice. The magnetic state cardinally changes as the concentration of niobium reaches $25 \%$. We have observed the magnetic susceptibility of the $x=0.5$ sample dramatically decreases. ZFC magnetization shows a peak at $30 \mathrm{~K}$. Below this temperature FC magnetization practically does not change. Taking into account the character of $M(H)$ dependence we have concluded that the sample $x=0.5$ can be considered as spin glass with $T_{f}=30 \mathrm{~K}$. We can explain the collapse of long range ferromagnetic ordering by a diamagnetic dilution of $\mathrm{Mn}$ - sublattice. According to resistivity versus temperature measurements $\mathrm{La}_{1-x} \mathrm{Sr}_{x}\left(\mathrm{Mn}_{1-x / 2} \mathrm{Nb}_{x / 2}\right) \mathrm{O}_{3}$ samples are semiconductors. Below Curie point a large value of magnetoresistance is observed.

The results presented here deal with the facts that the Nb-doped $\mathrm{La}_{1-x} \mathrm{Sr}_{x}\left(\mathrm{Mn}_{1-x / 2}^{3+} \mathrm{Nb}_{x / 2}^{5+}\right) \mathrm{O}_{3}$ samples enriched with $\mathrm{Mn}^{3+}$ ions are ferromagnetic and show a large magnetoresistance. It is worth noting that the possibility of the existence of ferromagnetic ordering in the manganites, despite the absence of $\mathrm{Mn}^{3+}$ ions, reject the double exchange and the electronic phase separation concepts. The result obtained indicates an important role of ferromagnetic superexchange via oxygen scenario of magnetic interactions in manganites. According to the superexchange mechanism the $\mathrm{Mn}^{3+}-\mathrm{O}-\mathrm{Mn}^{3+}$ and $\mathrm{Mn}^{3+}-\mathrm{O}-\mathrm{Mn}^{4+} 180^{\circ}$ magnetic interactions are strongly ferromagnetic for the orbitally 
disordered state whereas the $\mathrm{Mn}^{4+}-\mathrm{O}-\mathrm{Mn}^{4+}$ ones are strongly antiferromagnetic [4]. The Curie point associated with $\mathrm{Mn}^{3+}-\mathrm{O}-\mathrm{Mn}^{3+}$ positive superexchange may be close to room temperature for manganites with perovskite structure because our samples contain diamagnetic $\mathrm{Nb}^{5+}$ ions which should strongly decrease the Curie point. Stoichiometric $\mathrm{LaMn}^{3+} \mathrm{O}_{3}$ compound also shows ferromagnetic interactions between $\mathrm{Mn}^{3+}$ ions when cooperative Jahn-Teller distortions are vanished at $T=750 \mathrm{~K}$. The orbital ordering changes character of superexchange magnetic interactions which in the orbitally ordered state become anisotropic [4,28].

$$
\text { 2.3. } \mathrm{Nd}_{1-x} \mathrm{Ca}_{x} \mathrm{MnO}_{3} \text { system }
$$

The hypothetical magnetic phase diagram of the $\mathrm{Nd}_{1-x} \mathrm{Ca}_{x} \mathrm{MnO}_{3}$ system at low Ca doping level is presented in Fig. 4. Neutron diffraction shows that the samples with $x<0.08$ consist mainly of antiferromagnetic phase while at $x \geq 0.08$ ferromagnetic component dominates. Under hole doping the temperature of the transition into paramagnetic state at first decreases and then around $x=0.1$ increases. We have observed two magnetic phase transitions in the range $0.06 \leq x \leq 0.1$ as temperature decreases.

The $\mathrm{Nd}_{1-x} \mathrm{Ca}_{x} \mathrm{MnO}_{3}$ solid solutions contain two types of magnetically active sublattices: neodymium and manganese ones. At first we discuss the Nd contribution into magnetic properties. The $f-f$ exchange interaction in rare-earth sublattice is as a rule rather weak in comparison with $d-d$ interaction between manganese ions. One can expect that neodymium magnetic moments should order as a result of $f-d$ exchange interactions between neodymium and manganese sublattices. The study of magnetic properties of $\mathrm{Nd}_{1-x} \mathrm{Ca}_{x} \mathrm{MnO}_{3}$ samples confirm this viewpoint. According to neutron diffraction data the magnetic moments of neodymium ions start to be ordered slightly below $T_{N}$. Magnetic moment of $\mathrm{Nd}$ ion is about $1.2 \mu_{B}$ at $2 \mathrm{~K}$ and directed opposite to weak ferromagnetic vector in $\mathrm{NdMnO}_{3}$ while in the sample $x=0.12$ the orientation of $\mathrm{Nd}$ and $\mathrm{Mn}$ magnetic moments is the same. In the range $0.06 \leq x \leq 0.10$ the metamagnetic behavior was observed in large magnetic fields (triple hysteresis loops with a negative remanent magnetization).

According to our hypothesis, samples in the range $0.06 \leq x \leq 0.10$ consist of antiferromagnetic (weak ferromagnetic) and ferromagnetic phases which are exchange coupled at the boundary. The neodymium sublattice in both weak ferromagnetic and ferromagnetic phases orders nearby the Néel point (Curie point). However, the orientation of neodymium magnetic moments in both these phases is different: $f-d$

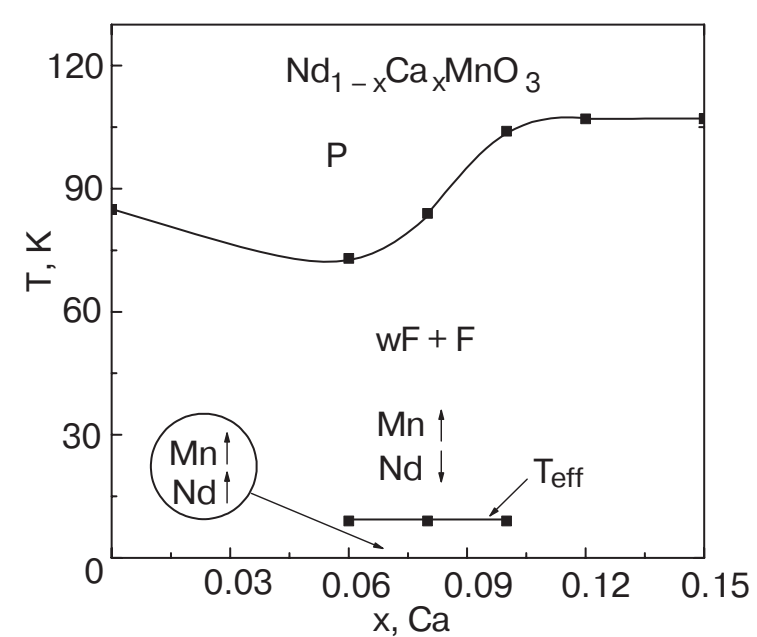

Fig. 4. Magnetic phase diagram of the $\mathrm{Nd}_{1-x} \mathrm{Ca}_{x} \mathrm{MnO}_{3}$ low-doped manganites: $\mathrm{wF}$ is weak ferromagnet, $\mathrm{F}$ is ferromagnet, $\mathrm{P}$ is paramagnet, $T_{\text {eff }}$ is effective temperature of the reorientational phase transition.

exchange is positive for ferromagnetic phase whereas it is negative in weak ferromagnetic phase. The ferromagnetic phase strongly affects magnetic properties of weak ferromagnetic phase due to exchange coupling at the boundary. This interaction may induce a reorientational transition from antiparallel orientation of neodymium moments and weak ferromagnetics vector to parallel one. We believe that nearby certain temperature the ground state of $\mathrm{Nd}^{3+}$ ions becomes degenerate because opposite contributions from exchange coupled ferromagnetic and weak ferromagnetic phases at Nd site become equal. According to theoretical consideration this state should be unstable thus leading to magnetic structure transformation [29]. Neutron diffraction study carried out for the $x=0.08$

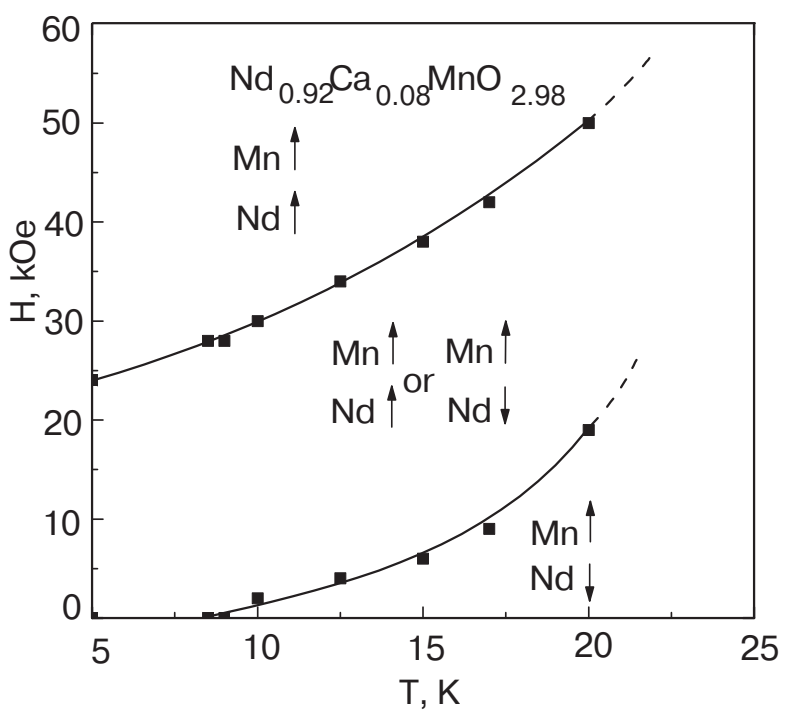

Fig. 5. The $H-T$ magnetic phase diagram for $\mathrm{Nd}_{0.92} \mathrm{Ca}_{0.08} \mathrm{MnO}_{2.98}$ compound. 
sample is in agreement with this interpretation of low temperature phase transition.

On the basis of magnetization data we propose $H-T$ magnetic phase diagram of $\mathrm{Nd}_{0.92} \mathrm{Ca}_{0.08} \mathrm{MnO}_{2.98}$ compound (Fig. 5). Depending on the prehistory in the wide range of magnetic field the phases with parallel or antiparallel orientation of neodymium and manganese sublattices in weak ferromagnetic phase can be realized. One can see that the value of magnetic field required for the change of relative orientation of the Nd and Mn magnetic moments in the weak ferromagnetic phase increases as temperature rises. The width of a field range in which the hysteresis is observed practically does not depend on a temperature. This type of magnetic phase diagram is in agreement with crossover of energy sub-levels of $\mathrm{Nd}$ ions.

$$
\text { 2.4. } \mathrm{Bi}_{1-x} \mathrm{Ca}_{x} \mathrm{MnO}_{3} \text { system }
$$

Figure 6 presents a magnetic phase diagram of the $\mathrm{Bi}_{1-x} \mathrm{Ca}_{x} \mathrm{MnO}_{3}$ manganites. As the calcium content in the $\mathrm{Bi}_{1-x} \mathrm{Ca}_{x} \mathrm{MnO}_{3}$ system increases, the latter passes through three different magnetic states, namely, ferromagnetic $(x \leq 0.1)$, spin-glass $(0.15 \leq x \leq 0.25)$, and antiferromagnetic $(x>0.25)$. In the case of antiferromagnetic compositions, the magnetic-ordering and structural-transformation temperatures vary only weakly within the concentration interval from $x=$ $=0.25$ to 0.6. The ferromagnetic ordering in $\mathrm{BiMnO}_{3}$ is most likely due to cooperative ordering of the $d_{x^{2}-y^{2}}$ orbitals [30,31]. With orbital ordering of this type, according to the Goodenough - Kanamori rules, ferromagnetic ordering becomes more energetically favorable than antiferromagnetic ordering. We may recall that rare-earth manganites exhibit orbital ordering of the $d_{z^{2}}$-type, which stabilizes the $A$-type antiferromagnetic structure [4]. Orbital disorder in $\mathrm{BiMnO}_{3}$ sets in, apparently, at a fairly high temperature, near $760 \mathrm{~K}$. Replacement of bismuth ions by calcium results in the formation of quadrivalent manganese ions, which should be accompanied by destruction of orbital ordering due to the appearance of nonJahn-Teller $\mathrm{Mn}^{4+}$ ions in the lattice. However, the orbitally disordered phase in manganites should be ferromagnetic $[4,28]$, whereas we observed a state of the spin-glass type. A direct transition from the antiferromagnetic to the spin-glass phase without passing through the ferromagnetic state was observed to occur in the rare-earth manganites $\mathrm{Sm}_{1-x} \mathrm{Ba}_{x} \mathrm{MnO}_{3}$ and $\mathrm{Y}_{1-x} \mathrm{Ca}_{x} \mathrm{MnO}_{3}(x \sim 0.12)$ [32,33]. It should be pointed out that at approximately this concentration of rare-earth ions, the ferromagnetic-spin glass transition takes place in $\mathrm{Bi}_{1-x} \mathrm{Ca}_{x} \mathrm{MnO}_{3}$.

There is more than one opinion on the nature of exchange interactions in manganites. The antiferromag-

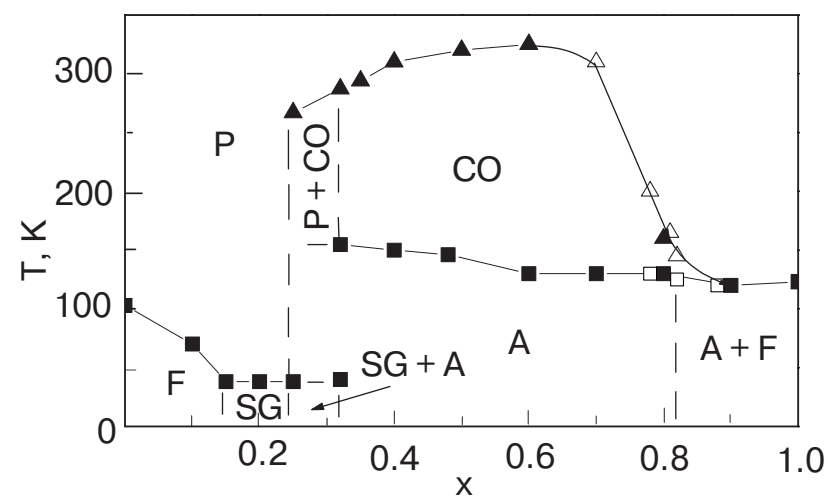

Fig. 6. Magnetic phase diagram of the $\mathrm{Bi}_{1-x} \mathrm{Ca}_{x} \mathrm{MnO}_{3}$ manganites: $\mathrm{A}$ is antiferromagnet, $\mathrm{F}$ is ferromagnet, $\mathrm{P}$ is paramagnet, $\mathrm{SG}$ is spin glass, $\mathrm{CO}$ is charge-ordered state.

netic state certainly forms through oxygen-mediated superexchange interactions of the type $\mathrm{Mn}-\mathrm{O}-\mathrm{Mn}$. Most researchers believe that the ferromagnetic state in manganites is created through double exchange, i.e., via direct carrier transfer between various lattice sites. In order for such an exchange mechanism to operate, manganese ions in different valence states must be present and the electrical conductivity must be high. The presence of manganese ions of different valencies is not a sufficient condition for high electrical conductivity; indeed, the $3 d$-orbitals of manganese and the $2 p$-orbitals of oxygen should also overlap strongly. It is believed that this parameter is controlled by the $\mathrm{Mn}-\mathrm{O}-\mathrm{Mn}$ bond angle [4,30]. The larger the lanthanide ion, the larger should be the $\mathrm{Mn}-\mathrm{O}-\mathrm{Mn}$ angle, the wider the $3 d$-band, and, accordingly, the higher the magnetic ordering temperature and the electrical conductivity. It was observed that the magnetic state of the manganites also depends on the difference between the ionic radii of the rare-earth and the lanthanide ions. A large difference between the radii lowers, as a rule, the magnetic ordering temperature as a result of competition between various exchange interactions characterized by a large difference in the $\mathrm{Mn}-\mathrm{O}-\mathrm{Mn}$ angles. This is why the spin-glass state sets in in the $\mathrm{Sm}_{1-x} \mathrm{Ba}_{x} \mathrm{MnO}_{3}$ system, wherein the average radius of the $\mathrm{Sm}$ and $\mathrm{Ba}$ ions is far larger than that between the $\mathrm{Y}$ and $\mathrm{Ca}$ cations in the $\mathrm{Y}_{1-x} \mathrm{Ca}_{x} \mathrm{MnO}_{3}$ system [32,33]. However, in all rareearth manganites, the $\mathrm{Mn}^{3+}-\mathrm{O}-\mathrm{Mn}^{4+}$ exchange coupling in the orbitally disordered phase is apparently ferromagnetic. The $\mathrm{Mn}-\mathrm{O}-\mathrm{Mn}$ angles in bismuthbased manganites are fairly large, which is supported by structural studies [31] and the quite high Curie temperature of $\mathrm{BiMnO}_{3}$. Hence, in the case of an orbitally disordered phase, one can expect the ferromagnetic part of exchange interactions to be dominant, which is at odds with experiment. Therefore, we be- 
lieve that, in contrast to the rare-earth manganites, no orbitally disordered phase forms in the $\mathrm{BiMnO}_{3}$ system in the concentration interval $0.1 \leq x \leq 0.3$. The spin-glass state forms in the $\mathrm{Bi}_{1-x} \mathrm{Ca}_{x} \mathrm{MnO}_{3}$ system most likely as a result of competition between ferromagnetic interactions in $\mathrm{BiMnO}_{3}$-type clusters and antiferromagnetic coupling in clusters in which the $\mathrm{Mn}^{3+}$ orbitals are frozen in random orientations. As the $\mathrm{Ca}^{2+}$ concentration increases, a new type of antiferromagnetic clusters, apparently due to charge ordering, appears. The existence in $\mathrm{Bi}_{0.75} \mathrm{Ca}_{0.25} \mathrm{MnO}_{3}$ of large clusters, charge-ordered in a similar way to those in $\mathrm{Bi}_{0.5} \mathrm{Ca}_{0.5} \mathrm{MnO}_{3}$, is suggested in studies of its elastic properties. Despite the presence of the spin-glass-type ground state, there is a certain fraction of states characterized by short-range order of the type of a charge-ordered phase, which is indicated by the fact that the Young modulus minima for the $x=0.25$ and 0.35 compositions are close in temperature. We believe that the extremely high stability of the orbitally and charge-ordered states in bismuth-based manganites derives from the strongly anisotropic character of the $\mathrm{Bi}-\mathrm{O}$ covalent bonding.

\section{Conclusions}

The magnetic and structural phase diagrams of $\mathrm{La}_{0.88} \mathrm{MnO}_{x}, \quad \mathrm{La}_{1-x} \mathrm{Sr}_{x}\left(\mathrm{Mn}_{1-x / 2} \mathrm{Nb}_{x / 2}\right) \mathrm{O}_{3}$, $\mathrm{Nd}_{1-x} \mathrm{Ca}_{x} \mathrm{MnO}_{3}$, and $\mathrm{Bi}_{1-x} \mathrm{Ca}_{x} \mathrm{MnO}_{3}$ manganites have been proposed. It has been shown that the magnetic properties of the samples under study are determined by the type of their orbital state. The dynamic correlations of $d_{3 z^{2}-r^{2}}$ orbitals favor ferromagnetic ordering in the manganites, while $A$-type antiferromagnetic structure is typical for the static Jahn-Teller distortions. It has been argued that concentrational transition from an antiferromagnetic to a ferromagnetic state occurs via the formation of inhomogeneous state due to structural phase separation mechanism.

This work was partly supported by Belarus and Ukrainian Fund for Basic Research (grant F04MS-004 and grant 10.01/001 Ф05K-012) Polish - Ukrainian Academies research project (No.4-2003) and by MES of Ukraine (grant M/257-2004).

1. C. Zener, Phys. Rev. 82, 403 (1951).

2. A.J. Millis, B.I. Shraiman, and R. Müller, Phys. Rev. Lett. 77, 175 (1996).

3. E.L. Nagaev, Phys. Rep. 346, 387 (2001).

4. J.B. Goodenough, A. Wold, R.J. Arnott, and N. Menyuk, Phys. Rev. 124, 373 (1961).

5. G.-L. Liu, J.-S. Zhou, and J.B. Goodenough, Phys. Rev. B64, 144414 (2001).
6. G. Biotteau, M. Hennion, F. Moussa, J. RodriguezCarvajal, L. Pinsard, A. Revcolevschi, Y.M. Mukovskii, and D. Shulyatev, Phys. Rev. B64, 104421 (2001).

7. G. Allodi, R. De Renzi, and G. Guidi, Phys. Rev. B57, 1024 (1998).

8. G. Papavassiliou, M. Fardis, M. Belesi, T.G. Maris, G. Kallias, M. Pissas, D. Niarchos, C. Dimitropoulos, and J. Dolinsek, Phys. Rev. Lett. 84, 761 (2000).

9. G. Papavassiliou, M. Belesi, M. Fardis, and C. Dimitropoulos, Phys. Rev. Lett. 87, 177204 (2001).

10. M.M. Savosta, V.I. Kamenev, V.A. Borodin, P. Novak, M. Marysco, J. Hejtmanek, K. Dorr, and M. Sahata, Phys. Rev. B67, 094403 (2003).

11. P.A. Algarabel, J.M. De Teresa, J. Blasco, M.R. Ibarra, Cz. Kapusta, M. Sikora, D. Zajac, P.C. Riedi, and C. Ritter, Phys. Rev. B67, 134402 (2003).

12. M. Hennion, F. Moussa, G. Biotteau, J. RodriguezCarvajal, L. Pinsard, and A. Revcolevschi, Phys. Rev. Lett. 81, 1957 (1998).

13. R.H. Heffner, L.P. Le, M.F. Hundley, J.J. Neumeier, G.M. Luke, K. Kojima, B. Nachumi, Y.J. Uemura, D.E. MacLaughlin, and S.-W. Cheong, Phys. Rev. Lett. 77, 1869 (1996).

14. C.H. Booth, F. Bridges, G.H. Kwei, J.M. Lawrence, A.L. Cornelius, and J.J. Neumeier, Phys. Rev. Lett. 80, 853 (1998).

15. M. Fath, S. Freisem, A.A. Menovsky, Y. Tomioka, J. Aarts, and J.A. Mydosh, Science 285, 1540 (1999).

16. M. Uehara, S. Mori, C.H. Chen, and S.-W. Cheong, Nature (London) 399, 560 (1999).

17. B.C. Hauback, H. Fjellvag, and N. Sakai, J. Solid State Chem. 124, 43 (1996).

18. T. Shibata, B. Bunker, J.F. Mitchel, and P. Schiffer, Phys. Rev. Lett. 88, 207205 (2002).

19. J. Rodriguez-Carvajal, M. Hennion, F. Moussa, A.H. Moudden, L. Pinsard, and A. Revcolevschi, Phys. Rev. B57, R3189 (1998).

20. M.C. Sanchez, G. Subias, J. Garcia , and J. Blasco, Phys. Rev. Lett. 90, 045503 (2003).

21. D. Louca, T. Egami, E.L. Brosha, H. Roder, and A.R. Bishop, Phys. Rev. B56, R8475 (1997).

22. T. Mizokawa, D.I. Khomskii, and G.A. Sawatzky, Phys. Rev. B63, 024403 (2001).

23. Y. Endoh, K. Hirota, S. Ishihara, S. Okamoto, Y. Murakami, A. Nishizawa, T. Fukuda, H. Kimura, H. Nojiri, K. Kaneko, and S. Maekawa, Phys. Rev. Lett. 82, 4328 (1999).

24. S. Okamoto, S. Ishihara, and S. Maekawa, Phys. Rev. B61, 14647 (2000).

25. R. Kajimoto, H. Mochizuki, and H. Yoshizawa, Physica B329-333, 738 (2003).

26. R.L. Rasera and G.L. Catchen, Phys. Rev. B58, 3218 (1998).

27. E.O. Wollan and W.C. Koehler, Phys. Rev. 100, 545 (1955).

28. J.B. Goodenough, J.-S. Zhou, F. Rivadulla, and E. Winkler, J. Solid State Chem. 175, 116 (2003). 
29. A.K. Zvezdin, V.M. Matveev, A.A. Mukhin, and A.A. Popov, Rare-Earth Ions in Magnetically Ordered Crystals, Science, Moscow (1985).

30. I.O. Troyanchuk, N.V. Kasper, O.S. Mantytskaya, and S.P. Pastushonok, Zh. Eksp. Teor. Fiz. 105, 239 (1994) [JETP 78, 212 (1994)].

31. T. Atou, H. Chiba, K. Ohoyama, Y. Yamaguchi, and Y. Syono, J. Solid State Chem. 145, 639 (1999).
32. I.O. Troyanchuk, D.D. Khalyavin, S.V. Truhanov, and H. Szymczak, J. Phys.: Condens. Matter 11, 8707 (1999).

33. R. Mathieu, P. Nordblad, D.N.H. Nam, N.X. Phuc, and N.V. Khiem, Phys. Rev. B63, 174405 (2001). 